\title{
Increased Succinate Accumulation Induces ROS Generation in In Vivo Ischemia/Reperfusion-Affected Rat Kidney Mitochondria
}

\author{
Justina Kamarauskaite, ${ }^{1,2}$ Rasa Baniene (D), ${ }^{1,3}$ Darius Trumbeckas, ${ }^{4}$ Arvydas Strazdauskas, ${ }^{1,3}$ \\ and Sonata Trumbeckaite ${ }^{1,2}$ \\ ${ }^{1}$ Neuroscience Institute, Lithuanian University of Health Sciences, Eiveniu g. 4, LT-50009 Kaunas, Lithuania \\ ${ }^{2}$ Department of Pharmacognosy, Medical Academy, Lithuanian University of Health Sciences, Sukileliu pr. 13, \\ LT-50009 Kaunas, Lithuania \\ ${ }^{3}$ Department of Biochemistry, Medical Academy, Lithuanian University of Health Sciences, Eiveniu g. 4, \\ LT-50009 Kaunas, Lithuania \\ ${ }^{4}$ Department of Urology, Medical Academy, Lithuanian University of Health Sciences, Eiveniu g. 2, LT-50009 Kaunas, Lithuania
}

Correspondence should be addressed to Rasa Baniene; rasa.baniene@lsmuni.lt

Received 1 September 2020; Revised 30 September 2020; Accepted 3 October 2020; Published 13 October 2020

Academic Editor: Erika Di Zazzo

Copyright (C) 2020 Justina Kamarauskaite et al. This is an open access article distributed under the Creative Commons Attribution License, which permits unrestricted use, distribution, and reproduction in any medium, provided the original work is properly cited.

\begin{abstract}
Mitochondria are recognized as main reactive oxygen species (ROS) producers, involving ROS generation by mitochondrial complexes I and III. Lately, the focus has been shifting to the ROS generation by complex II. Contribution of complex II (SDH) to ROS generation still remains debatable, especially in in vivo settings. Moreover, it is not completely defined at what time of ischemia the first alterations in mitochondria and the cell begin, which is especially important with renal arterial clamping in vivo during kidney surgery, as it predicts the postischemic kidney function. The aim of this study on an in vivo rat kidney ischemia/reperfusion model was to determine if there is a connection among (a) duration of kidney ischemia and mitochondrial dysfunction and (b) succinate dehydrogenase activity, succinate accumulation, and ROS generation in mitochondria at low and saturating succinate concentrations. Our results point out that (1) mitochondrial disturbances can occur even after 30 min of kidney ischemia/reperfusion in vivo and increase progressively with the prolonged time of ischemia; (2) accumulation of succinate in cytosol after ischemia/reperfusion correlated with increased $\mathrm{H}_{2} \mathrm{O}_{2}$ generation mediated by complex II, which was most noticeable with physiological succinate concentrations; and (3) ischemia/reperfusion induced cell necrosis, indicated by the changes in LDH activity. In conclusion, our new findings on the accumulation of succinate in cytosol and changes in SDH activity during kidney ischemia/reperfusion may be important for energy production after reperfusion, when complex I activity is suppressed. On the other hand, an increased activity of succinate dehydrogenase is associated with the increased ROS generation, especially with physiological succinate concentrations. All these observations play an important role in understanding the mechanisms which occur in the early phase of ischemia/reperfusion injury in vivo and may provide new ideas for novel therapeutic approaches or injury prevention; therefore, more detailed studies are necessary in the future.
\end{abstract}

\section{Background}

Ischemia/reperfusion (I/R) injury of the kidney is a complex process; it involves free radical overproduction, inflammation, disturbances in microcirculation, and damage of mitochondria, leading to postischemic kidney dysfunction [1]. Oxidative stress plays a central role in this process [2]. Mitochondria in the cell supply the energy in the form of ATP for basal cell functions as well as for cellular repair and regeneration; therefore, there is an emerging evidence about the importance of these organelles in I/R injury [3]. In previous studies, it has already been shown that $\mathrm{I} / \mathrm{R}$ leads to the reduced activity of mitochondrial respiratory chain complexes, ATP depletion, calcium accumulation, mitochondrial permeability transition pore opening, and an increase in reactive oxygen species (ROS) production; however, the 
complete mechanisms and the interaction between these processes are not fully elucidated. Moreover, it is not clear at what time of ischemia the first alterations in mitochondria and the cell begin, what duration of ischemia is already critical for the mitochondria, and how mitochondrial impairment progresses during ischemia. This is extremely important during renal surgery, when arteria is clamped for a certain period, as it determines the postischemic kidney recovery processes.

It is generally accepted that mitochondrial ROS generation is a potential contributory factor for kidney I/R injury and that ROS generation can be exacerbated during the postischemic reperfusion stage. Mitochondrial ROS not only drive acute damage but also initiate the pathology that develops over the minutes, days, and weeks following reperfusion. The mitochondrial respiratory chain complexes, namely, I and III, were considered as the main ROS producers under normal and pathological conditions [4, 5], while the contribution of complex II (SDH) was considered to be negligible [6]. However, in recent years, the impact of mitochondrial complex II in the generation of ROS has been getting more and more scientific interest. Chouchani et al. observed that ischemia in various organs, such as the brain, heart, liver, and kidney, induces accumulation of Krebs cycle intermediate succinate in mitochondria which was reoxidized by succinate dehydrogenase at reperfusion [7]. The authors proposed an interesting fact that the succinatedriven reverse electron transport through complex I was the main source of ROS under these conditions [7]. Quinlan et al., investigating skeletal muscle mitochondria, suggested that mitochondrial complex II generated superoxide radical in both, the forward and reverse reactions, and the authors also concluded that complex II could be a relevant producer of ROS under in vivo conditions such as hypoxia [8]. As far as we know, there are no data discussing whether kidney ische$\mathrm{mia} /$ reperfusion injury correlates with succinate accumulation and succinate dehydrogenase-induced ROS generation in vivo and whether it depends on the duration of ischemia in vivo.

The aim of this study on the in vivo rat kidney ischemia/reperfusion model was to determine if there is a connection among (a) duration of kidney ischemia and mitochondrial dysfunction and (b) succinate dehydrogenase activity, succinate accumulation, and ROS generation in mitochondria at low and saturating succinate concentrations.

\section{Materials and Methods}

2.1. Animals and Experimental Ischemia/Reperfusion In Vivo Model. The experimental procedures were performed according to the permission of the Lithuanian Committee of Good Laboratory Animal Use Practice (No. 0217/2011). Male Wistar rats (2-4 month-old), weighing 200-250 g, were housed under standard laboratory conditions, maintained on natural light and dark cycle and had free access to food and water. On the operation day, rats were anesthetized with a combination of an intraperitoneal injection of pentobarbital and ketamine. Pentobarbital $(200 \mathrm{mg} / \mathrm{mL})$ was diluted with isotonic $\mathrm{NaCl}$ solution $(0.9 \%)$ to a concentration of $20 \%$, and an intraperitoneal injection of $0.1 \mathrm{~mL} / 100 \mathrm{~g}$ rat weight was given. Ketamine $(100 \mathrm{mg} / \mathrm{kg})$ was administered intramuscularly $(0.1 \mathrm{~mL} / 100 \mathrm{~g}$ rat weight). Body temperature was kept constant at $37^{\circ} \mathrm{C}$ with a warming pad. A Yasargil clip (Aesculap, Tübingen, Germany) was placed over a rat's renal artery to induce renal normothermic ischemia $\left(37^{\circ} \mathrm{C}\right)$ for $20,30,40$, and $60 \mathrm{~min}$ with subsequent 30 minutes of reperfusion after removing the clip.

2.2. Preparation of Isolated Kidney Mitochondria. At the end of ischemia/reperfusion, the kidney was removed and placed into ice-cold medium containing $10 \mathrm{mM}$ Tris. $\mathrm{Cl}, 250 \mathrm{mM}$ sucrose, and $1 \mathrm{mM}$ EDTA ( $\mathrm{pH} 7.3$ ); the tissues were cut and homogenized in a glass-teflon homogenizer as described in [9]. The protein concentration was determined by the biuret method as described in [9]. Supernatants were collected and used for the determination of lactate dehydrogenase activities.

\subsection{Measurement of Kidney Mitochondrial Respiration.} Mitochondrial oxygen consumption rates was measured using Oroboros oxygraph-2 $\mathrm{k}$ in $2 \mathrm{~mL}$ incubation medium containing $10 \mathrm{mM}$ Tris. $\mathrm{Cl}, 5 \mathrm{mM} \mathrm{KH_{2 }} \mathrm{PO} 4,150 \mathrm{mM} \mathrm{KCl}$, $1 \mathrm{mM} \mathrm{MgCl}_{2} \times 6 \mathrm{H}_{2} \mathrm{O}, \mathrm{pH} 7.2,37^{\circ} \mathrm{C}$ with $5 \mathrm{mM}$ glutamate + $5 \mathrm{mM}$ malate, or $15 \mathrm{mM}$ succinate $+2 \mathrm{mM}$ amytal (an inhibitor of complex I) as respiratory substrates. All experimental conditions are described in [10]. The mitochondrial routine respiration rate $\left(V_{0}\right)$ was recorded in the medium supplemented with mitochondria and substrates (complex Idependent substrate: glutamate $5 \mathrm{mM}+$ malate $5 \mathrm{mM}$, or complex II-dependent substrate: succinate $15 \mathrm{mM}+2 \mathrm{mM}$ amytal as complex I inhibitor); original curves of mitochondrial respiration are seen in Figure 1. The effect of cytochrome $\mathrm{c}$ on respiration rate (indicating mitochondrial outer membrane permeability) was determined by adding $32 \mu \mathrm{M}$ cytochrome $\mathrm{c}\left(V_{3+\mathrm{cyt}}\right)$ to mitochondria respiring in state 3 . Then, the respiration was inhibited with $0.12 \mathrm{mM}$ atractyloside, an inhibitor of ADP/ATP translocator $\left(V_{\mathrm{ATR}}\right)$. Changes in atractyloside-inhibited respiration rates and routine respiration rates indicated the changes in the permeability of mitochondrial inner membrane. The respiratory control index (RCI) for glutamate/malate was calculated as the ratio of respiration rates $V_{\mathrm{ADP}} / V_{0}$ and for succinate as the ratio $V_{\mathrm{ADP}} / V_{\mathrm{ATR}}$. Cytochrome c effect was calculated as the ratio $\mathrm{V}_{\mathrm{ADP}+\mathrm{cyt} \mathrm{c}} / \mathrm{V}_{\mathrm{ADP}}$. Datlab 5 software (Oroboros Instruments) was used for real-time data acquisition and data analysis. Oxygen consumption rates were expressed as $\mathrm{pmol} / \mathrm{s} / 0.25 \mathrm{mg}$ mitochondrial protein.

2.4. Measurement of Mitochondrial Respiratory Chain Complexes I and II+III and Succinate Dehydrogenase Activity. The activity of complex I was measured spectrophotometrically at $340 \mathrm{~nm}$, according to the kinetics of NADH oxidation [9]. The activity of complex II+III in mitochondria was measured following the reduction of cytochrome $c$ at $550 \mathrm{~nm}$ (after the addition of succinate, respectively) using an extinction coefficient for cytochrome $c$ of $21.1 / \mathrm{mM} / \mathrm{cm}$ as described in [11]. Succinate dehydrogenase activity was measured at $600 \mathrm{~nm}$ following the reduction of 2,6- 

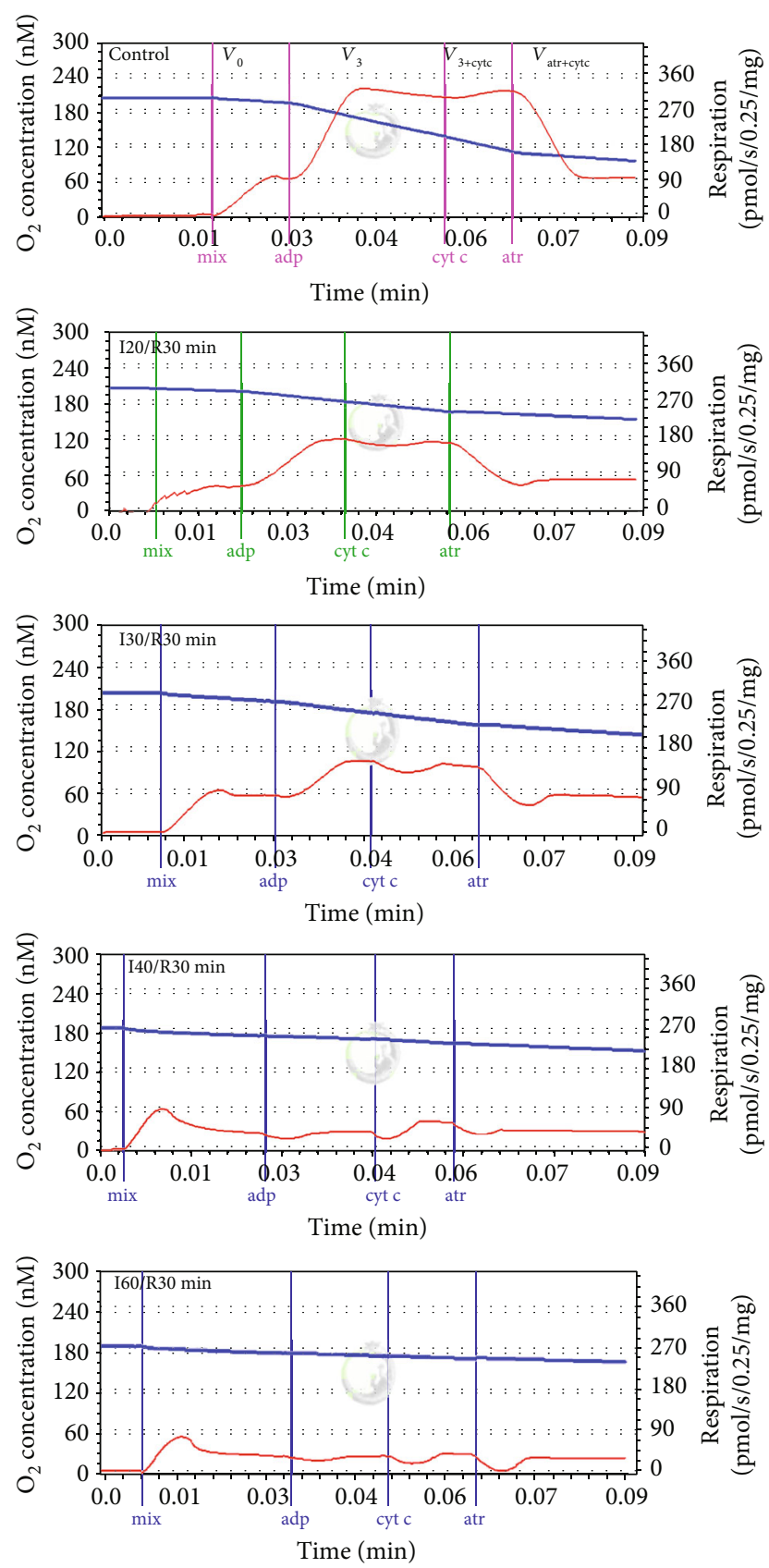

Figure 1: Original curves of kidney mitochondrial respiration in the control and ischemia/reperfusion (I/R) groups. Mitochondrial respiration rate was measured as described in Methods using $5 \mathrm{mM}$ glutamate plus $5 \mathrm{mM}$ malate as substrates. The blue trace represents oxygen concentration $(\mathrm{nmol} / \mathrm{mL})$, and the red trace represents oxygen flux. I20/R30, I30/R30, I40/R30, and I60/R30 refer to different ischemia durations (respectively, 20, 30, 40, and 60 minutes) following by 30 -minute reperfusion. $V_{0}$ : routine respiration rate in the presence of $0.5 \mathrm{mg} / \mathrm{mL}$ of mitochondria and substrates; $V_{3}$ : state 3 respiration rate in the presence of $1 \mathrm{mM}$ ADP; $V_{3+\text { cytc }}$ : state 3 respiration rate in the presence of $32 \mu \mathrm{M}$ cytochrome c; $V_{\mathrm{ATR}(+\mathrm{cytc})}$ : respiration rate in the presence of $0.12 \mathrm{mM}$ atractyloside and $32 \mu \mathrm{M}$ cytochrome c. dichlorophenolindophenol using an extinction coefficient of $19.1 / \mathrm{mM} / \mathrm{cm}$ as described in [12].

2.5. Measurement of Succinate Amount in Cytosolic and Mitochondrial Fractions. Lyophilized kidney cytosolic and mitochondrial fractions were used for chemical analysis. The samples $(10 \mathrm{mg})$ were dissolved in tetrahydrofuran ( $\geq 99.9 \%$ (HPLC) Sigma-Aldrich, Germany), transferred to a thermostatic ultrasound bath (Heidolph, Schwabach, Germany), and extracted for 15 minutes. The extracts were centrifuged for $5 \mathrm{~min}$ at $1699.36 \times \mathrm{g}$ (fixed angle rotor BRK5412, rotor type $12 \times 15 \mathrm{~mL}$ ). The supernatants obtained were removed from sediment, filtered through a Q-Max membrane filter $(25 \mathrm{~mm}$ in diameter, $0.45 \mu \mathrm{m}$ in pairs; Frisenette, Knebel, Denmark), and used for chromatographic analysis as indicated in [13].

2.6. Measurement of $\mathrm{H}_{2} \mathrm{O}_{2}$ Generation in Kidney Mitochondria. The generation of $\mathrm{H}_{2} \mathrm{O}_{2}$ in kidney mitochondria was determined fluorimetrically (fluorometer, Thermo Scientific) as described in [9]. Respiratory chain complex inhibitors: complex I inhibitor rotenone $(5 \mu \mathrm{M})$ and complex III inhibitor myxothiazol $(2 \mu \mathrm{M})$, were added to the appropriate wells. Fluorescence signal was calibrated using known amounts of $\mathrm{H}_{2} \mathrm{O}_{2}$.

2.7. Measurement of Lactate Dehydrogenase Activity. Lactate dehydrogenase (LDH) activity in cytosolic fractions was measured spectrophotometrically by monitoring NADH oxidation rate at $340 \mathrm{~nm}$ as described in [9].

2.8. Statistical Analysis. Data are presented as the mean \pm SEM of 4-11 separate experiments. The mean for individual experiment was obtained from at least three repetitive measurements. Statistical analysis was performed using the software package SPSS version 16.0 for Windows. $p<0.05$ was considered the level of significance.

\section{Results}

3.1. Changes in Kidney Mitochondrial Oxidative Respiration Rates after Postischemic Reperfusion. One of the consequences of ischemia/reperfusion induced injury is the suppression of mitochondrial respiration rates and activities of respiratory chain complexes. Our previous studies have revealed that kidney ischemia in vitro causes suppression of mitochondrial function in an ischemia-duration dependent manner [14]. Whether in vitro results could be extrapolated to in vivo experiments, the question remains open. Therefore, in this study, mitochondrial oxidative phosphorylation rates with two different substrates, complex I-dependent glutamate/malate and complex II-dependent succinate, first of all were tested in the in vivo kidney ischemia/ reperfusion model.

Figure 1 shows original mitochondrial respiratory curves in control and ischemia/reperfusion-affected mitochondria. Summary of results are presented in Table 1. Obvious changes were found in our study already after $30 \mathrm{~min}$ ischemia/reperfusion; i.e., mitochondrial state 3 respiration rate (in the presence of external ADP) was suppressed by $37 \%(p<0.05)$ as compared to control (i.e., nonischemic) 
TABLE 1: Effects of ischemia/reperfusion on kidney mitochondrial phosphorylation rates with glutamate/malate as substrates.

\begin{tabular}{lcccccc}
\hline & $V_{0(\mathrm{Glu})}$ & $V_{3(\mathrm{Glu})}$ & $V_{3+\text { cyt }(\mathrm{Glu})}$ & $V_{\text {ATR }(+\mathrm{cyt} \text { c })}$ & RCI $(\mathrm{Glu})$ & cyt c effect \\
\hline Control & $67 \pm 7$ & $210 \pm 17$ & $181 \pm 18$ & $75 \pm 6$ & $3.34 \pm 0.27$ & $0.87 \pm 0.04$ \\
I20/R30 min & $67 \pm 4$ & $205 \pm 19$ & $192 \pm 17$ & $71 \pm 6$ & $3.08 \pm 0.34$ & $0.94 \pm 0.03$ \\
I30/R30 min & $63 \pm 9$ & $132 \pm 9^{*}$ & $127 \pm 11^{*}$ & $56 \pm 7$ & $2.39 \pm 0.48$ & $0.96 \pm 0.04$ \\
I40/R30 min & $31 \pm 2^{*}$ & $50 \pm 5^{*}$ & $63 \pm 4^{*}$ & $32 \pm 1^{*}$ & $1.64 \pm 0.15^{*}$ & $1.30 \pm 0.12^{*}$ \\
I60/R30 min & $34 \pm 4^{*}$ & $30 \pm 4^{*}$ & $38 \pm 6^{*}$ & $26 \pm 4^{*}$ & $0.89 \pm 0.03^{*}$ & $1.26 \pm 0.03^{*}$ \\
\hline
\end{tabular}

Mitochondrial respiration rate was measured as described in Methods using $5 \mathrm{mM}$ glutamate plus $5 \mathrm{mM}$ malate as substrates. $V_{0}$ : routine respiration rate in the presence of $0.5 \mathrm{mg} / \mathrm{mL}$ of mitochondria and substrates; $V_{3}$ : state 3 respiration rate in the presence of $1 \mathrm{mM} \mathrm{ADP} ; V_{3+c y t c}$ : state 3 respiration rate in the presence of $32 \mu \mathrm{M}$ cytochrome c; $V_{\mathrm{ATR}(+\mathrm{cytc})}$ : respiration rate in the presence of atractyloside $0.12 \mathrm{mM}$ and $32 \mu \mathrm{M}$ cytochrome c. Mitochondrial respiratory control index (RCI), i.e., the ratio of oxygen uptake rate in state 3 to routine respiration rate $\left(\mathrm{RCI}=V_{3} / V_{0}\right)$. Cytochrome $\mathrm{c}$ effect (cyt $\mathrm{c}$ effect (Succ) $)$, i.e., the ratio of oxygen uptake rate in state 3 (in the presence of cytochrome c) to state 3 respiration rate (cyt c effect $\left.=V_{3+\text { cytc }} / V_{3}\right) .{ }^{*} p<0.05 v s$. control.

TABLE 2: Effects of ischemia/reperfusion on kidney mitochondrial phosphorylation rates with succinate as substrate.

\begin{tabular}{lcccccc}
\hline & $V_{0 \text { (Succ) }}$ & $V_{3 \text { (Succ) }}$ & $V_{3+\text { cyt c(Succ) }}$ & $V_{\text {ATR (+cyt c) }}$ & RCI (Glu) & cyt c effect \\
\hline Control & $153 \pm 20$ & $353 \pm 33$ & $382 \pm 34$ & $236 \pm 23$ & $2.49 \pm 0.27$ & $1.08 \pm 0.03$ \\
I20/R30 min & $229 \pm 21$ & $454 \pm 15^{*}$ & $506 \pm 21^{*}$ & $286 \pm 11$ & $2.04 \pm 0.20$ & $1.11 \pm 0.02$ \\
I30/R30 min & $206 \pm 17$ & $499 \pm 58^{*}$ & $633 \pm 85^{*}$ & $334 \pm 46$ & $2.39 \pm 0.12$ & $1.27 \pm 0.07^{*}$ \\
I40/R30 min & $262 \pm 35^{*}$ & $405 \pm 80$ & $677 \pm 97^{*}$ & $467 \pm 62^{*}$ & $1.47 \pm 0.15^{*}$ & $1.86 \pm 0.23^{*}$ \\
I60/R30 min & $71 \pm 15^{*}$ & $75 \pm 17^{*}$ & $266 \pm 26^{*}$ & $235 \pm 17^{*}$ & $1.05 \pm 0.04^{*}$ & $3.80 \pm 0.46^{*}$ \\
\hline
\end{tabular}

Mitochondrial respiration rate was measured as described in Methods using $15 \mathrm{mM}$ succinate $\left(+2 \mathrm{mM}\right.$ amytal) as substrate. $V_{0}$ : routine respiration rate in the presence of $0.5 \mathrm{mg} / \mathrm{mL}$ of mitochondria and substrates; $V_{3}$ : state 3 respiration rate in the presence of $1 \mathrm{mM} \mathrm{ADP} ; V_{3+c y t c}$ : state 3 respiration rate in the presence of $32 \mu \mathrm{M}$ cytochrome c; $V_{\mathrm{ATR}(+\mathrm{cyt})}$ : respiration rate in the presence of atractyloside $0.12 \mathrm{mM}$ and $32 \mu \mathrm{M}$ cytochrome c. Mitochondrial respiratory control index (RCI), i.e., the ratio of oxygen uptake rate in state 3 to routine respiration rate $\left(\mathrm{RCI}=V_{3} / V_{0}\right) .{ }^{*} p<0.05 v s$. control.

mitochondria (Table 1). After ischemia was prolonged to $40 \mathrm{~min}$ and $60 \mathrm{~min}$, a further decrease was observed in state 3 respiration rate (by $76 \%$ and $86 \%$, respectively) and in atractyloside-inhibited respiration rate $V_{\text {ATR }}$ (by $57 \%$ and $65 \%$, respectively), which shows changes in permeability of the inner mitochondrial membrane. The routine respiration $\left(V_{0}\right)$ rate decreased by $54 \%$ and $49 \%$, respectively $(p<0.05)$ (Table 1). As a consequence, the respiratory control index (RCI) also diminished by $30 \%, 51 \%$, and $73 \%(p<0.05)$ during longer ischemia/reperfusion periods ( $30 \mathrm{~min}, 40 \mathrm{~min}$, and 60 min) (Table 1). For instance, the RCI with glutamate/ malate as substrates after $60 \mathrm{~min}$ ischemia/reperfusion was found to be 0.89 , indicating full mitochondrial dysfunction.

In contrast, mitochondrial state 3 respiration with succinate (Table 2) as a substrate was drastically affected (decreased by $80 \%)$ only after a longer period $(60 \mathrm{~min})$ of ischemia/reperfusion, whereas during ischemia periods of $20 \mathrm{~min}, 30 \mathrm{~min}$, and $40 \mathrm{~min}$, it even caused a slight increase (of $29 \%, 41 \%(p<0.05)$, and $14 \%$, respectively) in state 3 respiration rates. The routine respiration rate $\left(V_{0}\right)$ as well as $V_{\text {ATR }}$ did not change statistically significantly after $20 \mathrm{~min}$ and $30 \mathrm{~min}$ of ischemia/reperfusion and increased by $71 \%$ and $97 \%$ after $40 \mathrm{~min}$ of ischemia/reperfusion $(p<0.05$, Table 2 ) causing a $41 \%$ decrease in RCI $(p<0.05$, Table 2$)$. As can be seen, when the ischemia period was prolonged to $60 \mathrm{~min}$, the mitochondrial function was fully disturbedthe RCI was found to be 1.05 , and there was no coupling between oxidation and phosphorylation. Additionally, we also checked the effect of ischemia/reperfusion on the intactness of mitochondrial outer membrane (after addition of exogenous cytochrome $c$ in state 3 ). The addition of cytochrome $\mathrm{c}$ had none or a slight effect on the intactness of mitochondrial outer membrane after a short (20 min and $30 \mathrm{~min}$ ) ischemia/reperfusion period, whereas after $40 \mathrm{~min}$ and $60 \mathrm{~min}$ of ischemia/reperfusion, it stimulated the state 3 respiration rate with both substrates, glutamate/malate, and succinate, but with succinate, it was much more obvious (respiration rate increased two and four times, respectively, $p<0.05$, Tables 1 and 2).

3.2. Kidney Ischemia/Reperfusion Affects the Complex I, Complex II+III, and Succinate Dehydrogenase Activity. Next, we investigated whether a decrease in glutamate/malate phosphorylation rates might be associated with the diminished activity of mitochondrial respiratory chain complexes. We revealed that even a short $(20 \mathrm{~min})$ ischemia/reperfusion period decreased complex I activity by $33 \%$ (Figure $2(\mathrm{a})$ ). Longer (30 $\mathrm{min}, 40 \mathrm{~min}$, and $60 \mathrm{~min}$ ) ischemia/reperfusion periods further reinforced (by 56\%, 62\%, and $85 \%, p<0.05$ ) these changes.

As the succinate-dependent respiration rate was increased after $20-40 \mathrm{~min}$ of ischemia/reperfusion, next, we measured the activity of complex II+III in kidney mitochondria. There were no changes in complex II+III activity after $20 \mathrm{~min}$ of ischemia/reperfusion, but it slightly increased after $30 \mathrm{~min}$ and $40 \mathrm{~min}$ of ischemia/reperfusion (by $33 \%$ and $44 \%$, 


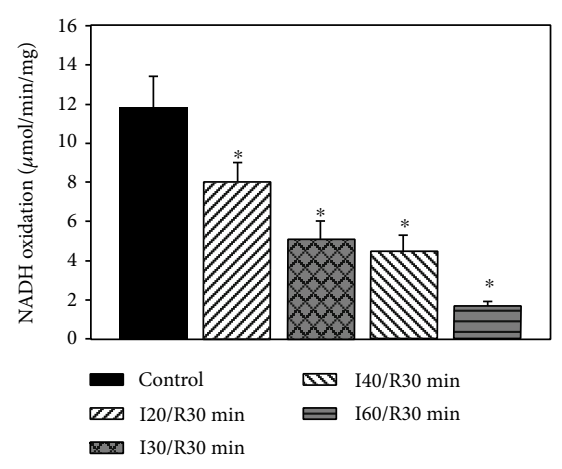

(a)

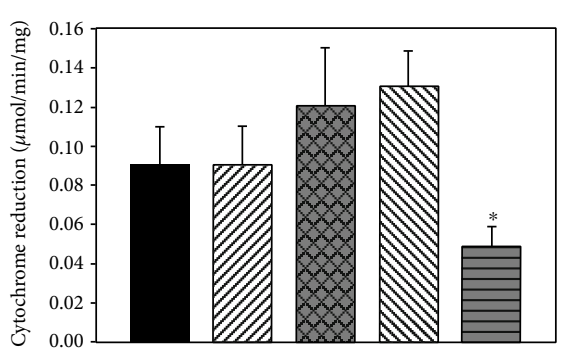

(b)

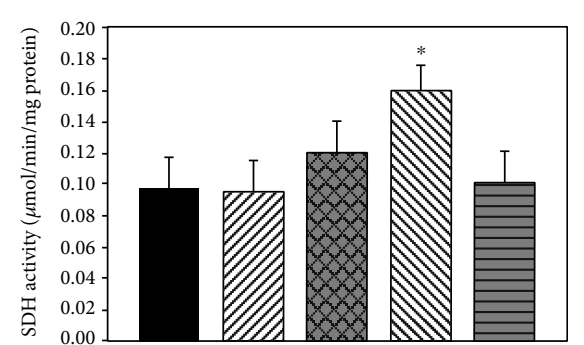

(c)

FIgURe 2: Effect of ischemia on complex I (a), complex II+III (b), and succinate dehydrogenase (c) activity in kidney mitochondria. The complex I activity was measured spectrophotometrically at $340 \mathrm{~nm}$ as described in Methods. ${ }^{*} p<0.05 v s$. control. The complex II activity was measured spectrophotometrically at $550 \mathrm{~nm}$ as described in Methods. ${ }^{*} p<0.05$ vs. control. The SDH activity was measured spectrophotometrically at $600 \mathrm{~nm}$ as described in Methods. ${ }^{*} p<0.05 v s$. control.

respectively) (Figures $2(\mathrm{~b}), p>0.05)$. After a longer (60 min) ischemia/reperfusion period, complex II+III activity decreased by $46 \%$ as compared with control $(p<0.05)$ and was supported by data of succinate respiration rate measurements. We also checked the activity of SDH and found that $20 \mathrm{~min}$ of ischemia/reperfusion had no effect on it (Figure 2(c)). When ischemia time was extended to $30 \mathrm{~min}$ and $40 \mathrm{~min}$, the activity of SDH increased by $23 \%(p>0.05)$ and by $65 \%$ $(p<0.05)$ as compared to control (nonischemic mitochondria), whereas after $60 \mathrm{~min}$ of ischemia/reperfusion, the SDH activity returned to the control level.

\subsection{Ischemia/Reperfusion Causes Succinate Accumulation in} Kidney Mitochondria. As in this study we found an increase in succinate respiration rate and in SDH activity after various periods of ischemia/reperfusion, next, we checked whether ischemia/reperfusion leads to accumulation of succinate in cytosolic and mitochondrial fractions. We found that after $30 \mathrm{~min}$ of ischemia/reperfusion, succinate concentration in the mitochondrial fraction increased by $15 \%$ as compared to control $(p<0.05)$ (Table 3), whereas the effect of $40 \mathrm{~min}$ ischemia/reperfusion on succinate content in mitochondria was similar to control (Table 3). Meanwhile, succinate content in the cytosolic fraction after $30 \mathrm{~min}$ ischemia/reperfusion increased more than 2 times as compared to control and continued to increase when ischemia time was prolonged to $40 \mathrm{~min}$ ischemia/reperfusion $(p<0.05)$ (Table 3$)$.

\subsection{Ischemia/Reperfusion Induces $\mathrm{H}_{2} \mathrm{O}_{2}$ Generation in} Kidney Mitochondria. Our new finding was that succinate concentration in the mitochondrial as well as in cytosolic fractions increased during certain periods of ischemia; therefore, we tested the hypothesis of its relationship with the increased ROS production in mitochondria. We revealed that $\mathrm{H}_{2} \mathrm{O}_{2}$ generation rate with glutamate + malate increased by $23 \%$ and $41 \%(p<0.05)$ after 30 and $40 \mathrm{~min}$ of ischemia/ reperfusion, respectively (Figure 3 ). The addition of complex I inhibitor rotenone significantly decreased $\mathrm{H}_{2} \mathrm{O}_{2}$ generation in control and in ischemia/reperfusion affected mitochondria (Figure 3(a)).
TABLE 3: Effect of ischemia/reperfusion on succinate content in the kidney ( $\mu \mathrm{g} / \mathrm{g}$ tissue).

\begin{tabular}{lcc}
\hline & $\begin{array}{c}\text { Succinate content in } \\
\text { mitochondria }\end{array}$ & $\begin{array}{c}\text { Succinate content } \\
\text { in cytosol }\end{array}$ \\
\hline Control group & $112.3 \pm 4.4$ & $8.1 \pm 1.4$ \\
I30/R30 min & $129.1 \pm 3.0^{*}$ & $19.2 \pm 2.0^{\#}$ \\
I40/R30 min & $114.9 \pm 2.2$ & $35.3 \pm 1.4^{\#}$ \\
\hline
\end{tabular}

Succinate concentration in mitochondria and cytosolic fractions was measured as described in Measurement of $\mathrm{H}_{2} \mathrm{O}_{2}$ Generation in Kidney Mitochondria. ${ }^{*} p<0.05$ vs. control; ${ }^{*} p<0.05$ vs. control.

The rate of $\mathrm{H}_{2} \mathrm{O}_{2}$ generation driven by saturating concentration of succinate $(5 \mathrm{mM})$ in control mitochondria was $0.78 \mathrm{nmol} / \mathrm{min} \mathrm{mg}$ protein, and this value was very similar to that induced by oxidation of glutamate + malate. $\mathrm{H}_{2} \mathrm{O}_{2}$ generation with $5 \mathrm{mM}$ concentration of succinate did not change significantly when ischemia time was prolonged to 30 and $40 \mathrm{~min}\left(\mathrm{H}_{2} \mathrm{O}_{2}\right)$ (Figure 3(b)). Rotenone inhibited $\mathrm{H}_{2} \mathrm{O}_{2}$ generation in control and ischemia/reperfusion affected mitochondria by $18 \%$ and $37 \%$, respectively $(p<0.05)$ (Figure $3(\mathrm{~b}))$. When rotenone and complex III inhibitor myxothiazol were added together, $\mathrm{H}_{2} \mathrm{O}_{2}$ generation rate after $30 \mathrm{~min}$ ischemia/reperfusion increased by $36 \%$ $(p<0.05)$ as compared with control mitochondria and by $31 \%(p<0.05)$ as compared with ROS generation when rotenone alone was added. When ischemia time was prolonged to $40 \mathrm{~min}, \mathrm{H}_{2} \mathrm{O}_{2}$ generation decreased and returned to the control level.

Next, we found that $\mathrm{H}_{2} \mathrm{O}_{2}$ generation rate in control and in ischemia/reperfusion ( $30 \mathrm{~min}$ and $40 \mathrm{~min}$ ) affected kidney mitochondria with subsaturating $(0.4 \mathrm{mM})$ succinate concentration (Figure 3(c)) was higher by $23 \%, 40 \%$, and $44 \%$, respectively $(p<0.05)$, as compared to saturating $(5 \mathrm{mM})$ succinate concentration. Thus, with a subsaturating succinate concentration, $30 \mathrm{~min}$ and $40 \mathrm{~min}$ ischemia/min reperfusion increased ROS generation by $35 \%(p>0.05)$ and $44 \%(p<0.05)$, respectively, as compared to the control group. The subsequent addition of rotenone significantly 


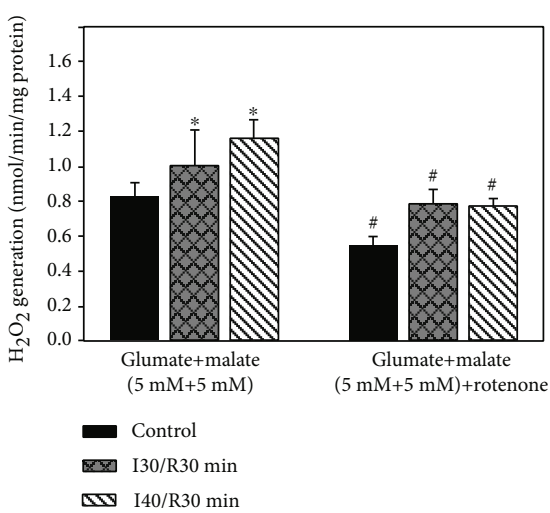

(a)

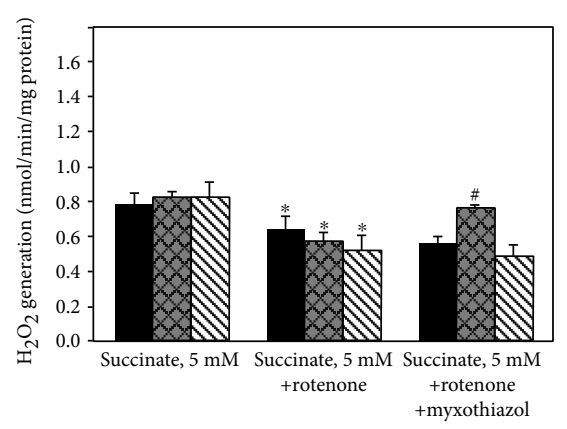

(b)

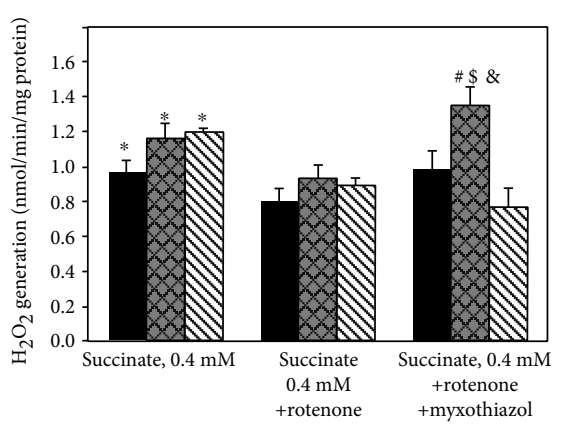

(c)

FIGURE 3: Effect of ischemia on $\mathrm{H}_{2} \mathrm{O}_{2}$ generation in kidney mitochondria with glutamate/malate (a) and succinate: saturating (b) and unsaturating (c) concentrations. The generation of $\mathrm{H}_{2} \mathrm{O}_{2}$ in kidney mitochondria was determined fluorimetrically as described in Methods (excitation at $544 \mathrm{~nm}$, emission at $590 \mathrm{~nm}$ ). Substrates: (a) glutamate $(5 \mathrm{mM})+$ malate $(5 \mathrm{mM})$; (b) succinate $5 \mathrm{mM}$; (c) succinate $0.4 \mathrm{mM}$. Respiratory chain complex inhibitors: rotenone $(5 \mu \mathrm{M})$ and myxothiazol $(2 \mu \mathrm{M}) .{ }^{*} p<0.05$ vs. succinate $5 \mathrm{mM}$ group; ${ }^{\#} p<0.05$ vs. control group; ${ }^{\&} p<0.05$ vs. succinate $5 \mathrm{mM}$ group; ${ }^{\lambda} p<0.05$ vs. control group; ${ }^{\$} p<0.05$ vs. succinate $0.4 \mathrm{mM}$ group; ${ }^{\phi} p<0.05$ vs. succinate $5 \mathrm{mM}$, with rotenone group; ${ }^{\S} p<0.05$ vs. succinate $5 \mathrm{mM}$, with rotenone and myxothiazol group; ${ }^{\psi} p<0.05$ vs. control group; ${ }^{\kappa} p<0.05$ vs. succinate $0.4 \mathrm{mM}$, with rotenone group; ${ }^{\alpha} p<0.05$ vs. succinate $5 \mathrm{mM}$, with rotenone and myxothiazol group.

decreased $\mathrm{H}_{2} \mathrm{O}_{2}$ generation by $16 \%$ and $26 \%$, in control and ischemia/reperfusion-affected mitochondria, respectively, as compared to the group without rotenone (Figure 3(c)). When rotenone and myxothiazol were added together, no effect on $\mathrm{H}_{2} \mathrm{O}_{2}$ generation was observed in control mitochondria, but it increased significantly after $30 \mathrm{~min}$ ischemia/ reperfusion (Figure 3(c)). ROS generation after $30 \mathrm{~min}$ ische$\mathrm{mia} /$ reperfusion was $43 \%$ higher as compared to the control group, $47 \%$ higher as compared to the group when rotenone alone was added, and $77 \%$ higher as compared to the group with saturating succinate concentrations. The ischemia prolonged from $30 \mathrm{~min}$ to $40 \mathrm{~min}$ decreased $\mathrm{H}_{2} \mathrm{O}_{2}$ generation by $43 \%$ as compared to the shorter ischemia/reperfusion group.

3.5. Ischemia/Reperfusion Increases Lactate Dehydrogenase (LDH) Activity in Cytosolic Fraction. LDH activity in the cytosolic fraction was measured as an indicator for tissue necrosis. In the cytosolic fraction of control kidneys, LDH activity was $1.48 \pm 0.1 \mathrm{IU} / \mathrm{min}$ per $\mathrm{mg}$ protein. After $20 \mathrm{~min}$ and $30 \mathrm{~min}$ ischemia/30 min reperfusion, there was no significant change in LDH activity. LDH activity increased about 2 times after $40 \mathrm{~min}$ ischemia/min reperfusion and about 1.5 times after $60 \mathrm{~min}$ ischemia/min indicating severe damage of kidney tissue (Figure 4).

\section{Discussion}

Kidney ischemia/reperfusion injury is unavoidable during kidney surgery and depending on duration of ischemia which may affect kidney function with different severities of consequences. Many studies on effects of ischemia are performed in vitro using animal models, but it is always important to evaluate and compare the changes that occur in mitochondria in vivo. Furthermore, in this study, we determined if there is a connection among (a) duration of kidney ischemia

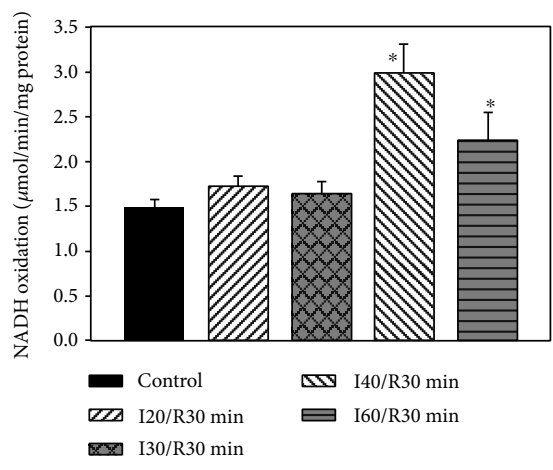

FIGURE 4: Effect of kidney ischemia on LDH activity in cytosolic fraction. LDH activity was measured as described in Methods. ${ }^{*} p<0.05$ vs. control.

and mitochondrial dysfunction and (b) succinate dehydrogenase activity, succinate accumulation, and ROS generation in mitochondria at low and saturating succinate concentrations.

In order to determinate changes that occur during in vivo ischemia/reperfusion injury, first, we measured the mitochondrial respiration rates with both glutamate/malate and succinate as mitochondrial substrates. Our results showed that mitochondrial phosphorylation rates with glutamate/ malate decreased even after a short time $(30 \mathrm{~min})$ ischemia/ reperfusion, and it was supported by the decreased activity of complex I. The prolongation of the ischemia period to $40 \mathrm{~min}$ and $60 \mathrm{~min} /$ reperfusion further reinforced mitochondrial damage (mitochondrial respiratory rates decreased by $86 \%$ and complex I activity by $85 \%$ ). This study is in agreement with our results of the in vitro ischemia model [14] which already showed the disturbances in glutamate/malate respiration rates after $20 \mathrm{~min}$ and even more so after a longer (40 $\mathrm{min}$ and $60 \mathrm{~min}$ ) ischemia period. Our in vivo study also revealed the damage of mitochondrial outer membrane and lack of cytochrome $\mathrm{c}$ after $30 \mathrm{~min}$ of ischemia/reperfusion 
(cytochrome c test), which increased drastically (two and four times) after $40 \mathrm{~min}$ and $60 \mathrm{~min}$ of ischemia/reperfusion respiring on succinate and supported our results in vitro [14]. However, our in vivo study revealed quite opposite results in terms of succinate dependent phosphorylation rates as compared to the in vitro model. We observed an increase in the succinate respiration rate after $20 \mathrm{~min}$ of ischemia/reperfusion, with a further increase after a prolonged (up to $40 \mathrm{~min}$ ) period of ischemia. This result led us to test hypothesis and to measure complex II+III and SDH activity as well as succinate amount in mitochondrial and cytosolic fractions at various points of ischemia/reperfusion. Our quite new finding is that succinate concentration in the cytosolic fraction increased several times ( 2 and 4 times) after $30 \mathrm{~min}$ and $40 \mathrm{~min}$ of ischemia/reperfusion. Thus, it indicated accumulation of succinate in cytosol that occurred during the ischemia/reperfusion period. Moreover, our unpublished results revealed that after $30 \mathrm{~min}$ of ischemia alone, succinate concentration in the mitochondrial fraction increased 1.40 times and in the cytosolic fraction about 3 times. These results also indicate a damaging effect of ischemia on mitochondrial integrity. Remembering that succinate-dependent phosphorylation rates increased by $29 \%, 41 \%(p<0.05)$, and $14 \%(p>0.05)$ after $20 \mathrm{~min}$, $30 \mathrm{~min}$, and $40 \mathrm{~min}$ of ischemia/reperfusion, respectively, and the activity of complexes II+III and SDH increased by $20 \%$ and $65 \%$ after $30 \mathrm{~min}$ and $40 \mathrm{~min}$ of ischemia/reperfusion, (Figures 2(b) and 2(c)), it seems that the increase in the activity of these complexes can help to maintain the mitochondrial proton motive force during reperfusion, especially in the case when complex I activity decreased drastically, for instance, after $30 \mathrm{~min}$ and $40 \mathrm{~min}$ of ischemia/reperfusion (Figure 2(a)). Our data showed that prolonged ischemia (60 min ischemia/reperfusion) induced a significant decrease in complexes II+III and SDH activity (by $46 \%$ and $38 \%$, respectively) (Figures $2(\mathrm{~b})$ and $2(\mathrm{c})$ ), as well as in succinate oxidation (by $80 \%$ ) (Table 2). It is interesting to note that Gonzalez-Flecha and Boveris revealed that in kidney submitochondrial particles from the cortex, $30 \mathrm{~min}$ and $60 \mathrm{~min}$ ischemia had no effect on SDH activity and only a slight (about 15\%) decrease of SDH activity after $10 \mathrm{~min}$ of reperfusion [15]. Moreover, our results on the increased activity of complexes II+III and SDH during certain periods of I/R were supported by data of the increased succinate accumulation after ischemia/reperfusion. Similar results were obtained by Andrienko et al.; they found that after $30 \mathrm{~min}$ of ischemia succinate concentration in a rat heart increased many times [16], and oxidation of succinate maintained the mitochondrial ATP synthesis. Therefore, to further confirm our hypothesis about the role of succinate in ROS production during ischemia/reperfusion injury, we investigated the relationship between the activity of $\mathrm{SDH}$, succinate accumulation, and production of ROS; it is generally acknowledged that ischemia/reperfusion-induced mitochondrial dysfunction might be associated with overproduction of ROS, generated by several mechanisms. It was shown by others that complexes I and II can produce ROS into the matrix and complex III can produce ROS on both sides of the mitochondrial inner membrane [17]. Recently, it was shown that ROS generation driven by complex II is especially important under pathological conditions when different parts of the respiratory chain can be inhibited [18], and therefore, succinate accumulation (which arises in ischemia from reversal of succinate dehydrogenase) can be associated with mitochondrial ROS production during reperfusion [19, 20]. This conclusion was supported by our present results. Thus, we revealed that accumulation of succinate in the cytosol after ischemia/reperfusion correlates with the increased $\mathrm{SDH}$ activity and $\mathrm{H}_{2} \mathrm{O}_{2}$ generation (Figures 2(b), 2(c), and 3(c)). $\mathrm{H}_{2} \mathrm{O}_{2}$ generation mediated by $\mathrm{SDH}$ (after addition of rotenone and myxothiazol) was the most noticeable with physiological succinate concentration after $30 \mathrm{~min}$ of ischemia/reperfusion (Figure 3(c)). It is important to note that for measurement of ROS generation during ischemia/reperfusion most of studies used only saturating concentrations of succinate. This is not sufficient for understanding the changes that occur under in vivo conditions. In the present study, we used physiological $(0.4 \mathrm{mM})$ succinate concentrations in order to detect changes that occur under in vivo conditions in ischemia/reperfusion and revealed the role of SDH in ROS generation during kidney ischemia/reperfusion. Chouchani et al. [7] observed the accumulation of succinate after ischemia and noticed that ischemic succinate accumulation was responsible for mitochondrial ROS production during reperfusion through a reverse electron transport at mitochondrial complex I [7]. Thus, our results indicate that ROS generation by reverse electron transport driven by complex I in kidney mitochondria is only one of several pathways. SDH, as was shown in our study, is also involved in ROS production, especially at physiological succinate concentrations (Figure 3(c)), and this is in line with Quinlan et al.'s observation that ROS production at complex II in muscle mitochondria occurs at low concentration of succinate when the succinate-binding site is not occupied by substrate and that ROS generation is inhibited by excess of succinate [8].

Furthermore, ischemia-induced accumulation of succinate in the cytosol and ROS generation (Table 3, Figures 3(b) and $3(\mathrm{c})$ ) mediates inflammatory response leading to cell death. The 2 and 1.5 times increased LDH activity after $40 \mathrm{~min}$ and $60 \mathrm{~min}$ of ischemia/reperfusion, an indicator for necrosis, was observed in our study and was in line with other studies (Figure 4) [14, 21, 22]. It is important to know that strict regulation of ROS levels is crucial for cellular life. In the cell, $\mathrm{H}_{2} \mathrm{O}_{2}$ plays the role of a second messenger and is involved in the control of gene expression and contribute to the control of cell proliferation and differentiation [23]. On the other hand, a moderate increase of ROS contributes to several pathologic conditions, among which are ischemia-reperfusion, inflammation, tumor promotion, and progression. So the development of therapeutic strategies which modulate mitochondrial and cellular ROS levels can be used for disease prevention or treatment [23].

In summary, our results point out that (1) mitochondrial disturbances can occur even after 30 min of kidney ischemia/reperfusion in vivo and increase progressively with the prolonged time of ischemia; (2) accumulation of succinate in cytosol after ischemia/reperfusion correlated 
with increased $\mathrm{H}_{2} \mathrm{O}_{2}$ generation mediated by complex II, which was most noticeable with physiological succinate concentrations; and (3) ischemia/reperfusion induced cell necrosis, indicated by the changes in LDH activity.

\section{Conclusion}

Thus, our new findings on the accumulation of succinate in cytosol and changes in SDH activity during kidney ischemia/reperfusion may be important for energy production after reperfusion, when complex I activity is suppressed. On the other hand, an increased activity of succinate dehydrogenase is associated with the increased ROS generation, especially with physiological succinate concentrations. All these observations play an important role in understanding the mechanisms which occur in the early phase of ischemia/ reperfusion injury in vivo and may provide new ideas for novel therapeutic approaches or injury prevention; therefore, more detailed studies are necessary in the future.

\section{Abbreviations}

I/R: Ischemia/reperfusion

LDH: Lactate dehydrogenase

RCI: Respiratory control index

SDH: Succinate dehydrogenase

ROS: Reactive oxygen species.

\section{Data Availability}

The datasets used and analyzed during the current study are available from the corresponding author on reasonable request.

\section{Ethical Approval}

The experimental procedures were performed according to the permission of the Lithuanian Committee of Good Laboratory Animal Use Practice (No. 0217/2011).

\section{Conflicts of Interest}

The authors declare that they have no competing interests.

\section{Authors' Contributions}

JK performed the experiments and analyzed the data. AS contributed the reagents or materials and interpreted the data. RB and ST designed the experiments, authored or reviewed the drafts of the paper, and approved the final draft. DT reviewed the drafts of the paper. All authors read and approved the final manuscript.

\section{References}

[1] J. M. Forbes, "Mitochondria-players in Function?," Trends in Endocrinology and Metabolism, vol. 27, no. 7, pp. 441-442, 2016.

[2] J. L. Martin, A. V. Gruszczyk, T. E. Beach, M. P. Murphy, and K. Saeb-Parsy, "Mitochondrial mechanisms and therapeutics in ischaemia reperfusion injury," Pediatric Nephrology, vol. 34, no. 7, pp. 1167-1174, 2019.

[3] E. J. Lesnefsky, Q. Chen, B. Tandler, and C. L. Hoppel, "Mitochondrial dysfunction and myocardial ischemia-reperfusion: implications for novel therapies," Annual Review of Pharmacology and Toxicology, vol. 57, no. 1, pp. 535-565, 2017.

[4] R. S. Balaban, S. Nemoto, and T. Finkel, "Mitochondria, oxidants, and aging," Cell, vol. 120, no. 4, pp. 483-495, 2005.

[5] P. Korge, S. A. John, G. Calmettes, and J. N. Weiss, "Reactive oxygen species production induced by pore opening in cardiac mitochondria: the role of complex II," The Journal of Biological Chemistry, vol. 292, no. 24, pp. 9896-9905, 2017.

[6] M. P. Murphy, "How mitochondria produce reactive oxygen species," The Biochemical Journal, vol. 417, no. 1, pp. 1-13, 2009.

[7] E. T. Chouchani, V. R. Pell, E. Gaude et al., "Ischaemic accumulation of succinate controls reperfusion injury through mitochondrial ROS," Nature, vol. 515, no. 7527, pp. 431-435, 2014.

[8] C. L. Quinlan, A. L. Orr, I. V. Perevoshchikova, J. R. Treberg, B. A. Ackrell, and M. D. Brand, "Mitochondrial complex II can generate reactive oxygen species at high rates in both the forward and reverse reactions," The Journal of Biological Chemistry, vol. 287, no. 32, pp. 27255-27264, 2012.

[9] G. Baliutyte, R. Baniene, S. Trumbeckaite, V. Borutaite, and A. Toleikis, "Effects of Ginkgo biloba extract on heart and liver mitochondrial functions: mechanism(s) of action," Journal of Bioenergetics and Biomembranes, vol. 42, no. 2, pp. 165-172, 2010.

[10] S. Trumbeckaite, N. Pauziene, D. Trumbeckas, M. Jievaltas, and R. Baniene, "Caffeic ester Ischemia-Inducedkidney injury in Rats," Oxidative Medicine and Cellular Longevity, vol. 2017, 11 pages, 2017.

[11] H. D. Tisdale, "Preparation and properties of succinic-cytochrome c reductase (complex II-III)," Methods in Enzymology, pp. 213-215, 1967.

[12] O. Arrigoni and T. P. Singer, "Limitations of the Assay for Succinic and Related Dehydrogenases," Nature, vol. 193, no. 4822, pp. 1256-1258, 1962.

[13] L. Jarukas, J. Kamarauskaitè, M. Marksa, S. Trumbeckaite, R. Banienè, and L. Ivanauskas, "Bio-based succinic acid sample preparation and derivatization procedure optimisation for gas chromatography-mass spectrometry analysis," ScienceRise: Pharmaceutical Science, vol. 4, no. 14, pp. 9-13, 2018.

[14] R. Baniene, D. Trumbeckas, M. Kincius et al., "Short ischemia induces rat kidney mitochondria dysfunction," Journal of Bioenergetics and Biomembranes, vol. 48, no. 1, pp. 77-85, 2016.

[15] B. Gonzalez-Flecha and A. Boveris, "Mitochondrial sites of hydrogen peroxide production in reperfused rat kidney cortex," Biochimica et Biophysica Acta (BBA) - General Subjects, vol. 1243, no. 3, pp. 361-366, 1995.

[16] T. N. Andrienko, P. Pasdois, G. C. Pereira, M. J. Ovens, and A. P. Halestrap, "The role of succinate and ROS in reperfusion injury - a critical appraisal," Journal of Molecular and Cellular Cardiology, vol. 110, pp. 1-14, 2017.

[17] R. Z. Zhao, S. Jiang, L. Zhang, and Z. Bin Yu, "Mitochondrial electron transport chain, ROS generation and uncoupling (review)," International Journal of Molecular Medicine, vol. 44, no. 1, pp. 3-15, 2019.

[18] L. Tretter, A. Patocs, and C. Chinopoulos, "Succinate, an intermediate in metabolism, signal transduction, ROS, hypoxia, 
and tumorigenesis," Biochimica et Biophysica Acta (BBA) General Subjects, vol. 1857, no. 8, pp. 1086-1101, 2016.

[19] P. V. Sahni, J. Zhang, S. Sosunov et al., "Krebs cycle metabolites and preferential succinate oxidation following neonatal hypoxic-ischemic brain injury in mice," Pediatric Research, vol. 83, no. 2, pp. 491-497, 2018.

[20] V. R. Pell, E. T. Chouchani, C. Frezza, M. P. Murphy, and T. Krieg, "Succinate metabolism: a new therapeutic target for myocardial reperfusion injury," Cardiovascular Research, vol. 111, no. 2, pp. 134-141, 2016.

[21] C. Philipponnet, J. Aniort, C. Garrouste, J. L. Kemeny, and A. E. Heng, "Ischemia reperfusion injury in kidney transplantation: a case report," Medicine (Baltimore), vol. 97, no. 52, p. e13650, 2018

[22] B. J. Padanilam, "Cell death induced by acute renal injury:a perspective on the contributions of apoptosis and necrosis," American Journal of Physiology-Renal Physiology, vol. 284, no. 4, pp. F608-F627, 2003.

[23] B. Perillo, M. di Donato, A. Pezone et al., "ROS in cancer therapy: the bright side of the moon," Experimental \& Molecular Medicine, vol. 52, no. 2, pp. 192-203, 2020. 